\title{
FLIM Analysis of Intracellular Markers Associated With the Development of Parkinson's Disease in Cellular Model
}

\author{
M. POKUSA ${ }^{1,2}$, A. KRÁL'OVÁ TRANČÍKOVÁ ${ }^{1,2}$ \\ ${ }^{1}$ Biomedical Center Martin, Jessenius Faculty of Medicine in Martin, Comenius University in \\ Bratislava, Martin, Slovakia, ${ }^{2}$ Department of Pathophysiology, Jessenius Faculty of Medicine in \\ Martin, Comenius University in Bratislava, Martin, Slovakia
}

Received March 28, 2018

Accepted September 17, 2018

\begin{abstract}
Summary
Parkinson's disease (PD) is currently the second most common neurodegenerative disorder in the world. Major features of cell pathology of the disease include the presence of cytoplasmic inclusions called Lewy bodies, which are composed of aggregated proteins. The presence of Lewy's body is associated with more advanced stages of the disease when considering irreversible changes. Precise identification of the disease stage at a cellular level presents the critical tool in developing early diagnostics and/or prevention of PD. The aim of our work is to introduce sensitive microscopic analysis in living cells, focused on initial intracellular changes and thus capable to detect earlier stages of the disease.
\end{abstract}

\section{Key words}

Fluorescence lifetime imaging • FLIM • SH-SY5Y • Cellular model - Rotenone • Parkinson's disease

\section{Corresponding author}

A. Král'ová Trančíková, Biomedical center Martin, Jessenius Faculty of Medicine in Martin, Comenius University in Bratislava, Malá hora 4D, Martin, 03601 , Slovakia. E-mail: alzbeta.trancikova@jfmed.uniba.sk

\section{Introduction}

Parkinson's disease is a progressive neurodegenerative disorder that is generally manifested by motor symptoms associated with degeneration of CNS dopaminergic neural pathways, as well as earlier manifestations of non-motor symptoms mainly related to the function of the enteric nervous system (Simuni and
Sethi 2008, Dickson et al. 2009). Slow progression of PD was already characterized by Braak, who noted, that progressive neurodegenerative changes at lower levels of the nervous system precede terminal stages of the disease (Braak et al. 2003).

One of the most typical manifestations of PD is the presence of cytoplasmic inclusions called Lewy body (LB), identified at various stages of PD in the CNS as well as in certain areas of GIT (Pan-Montojo et al. 2010, Nakamura et al. 2016).

Overexpression of $\alpha$-synuclein, the main protein found in LB, is considered to be a risk factor for many neuronal processes together with its accumulation observed closely to lipid bilayers (Iyer et al. 2014). Clear cytotoxicity of pre-expressed $\alpha$-synuclein and its protofibrils has been consistently documented by many studies (Wong and Krainc 2017), where it affects autophagic processes and membrane dynamics of almost all vesicular organelles (Papáčkova and Cachová 2014). The most studied structures are Golgi, ER with no exception for mitochondria (Hardy et al. 2006, Nakamura et al. 2008).

The disturbance of the mitochondrial membrane dynamics caused by $\alpha$-synuclein-induced inhibition of mitochondrial complex I has already been confirmed (Devi et al. 2008). Subsequent dysregulation of $\mathrm{NADH} / \mathrm{NADPH}$ metabolism related to increased oxidative stress has been recognized as an important characteristic of neurodegenerative diseases in addition to many metabolic disorders (Vokurková et al. 2015, Chouchani et al. 2014, Blacker and Duchen 2016). In experimental conditions, an inhibition of mitochondrial 
complex I is extensively used to simulate PD in animal and cellular models. One of the most commonly used compound is rotenone (Blesa and Przebodborski 2014, Hisahara and Shimohama 2011), which usually leads to $\alpha$-synuclein intracellular accumulation (Pan-Montojo et al. 2010). Other classical effects of rotenone associated with inhibition of complex I and thus reduced oxidation rate is accumulation of NADH. This is evident already few minutes after cell exposure to the inhibitor (Blacker et al. 2014).

$\mathrm{NADH}$, in contrast to its oxidized form of $\mathrm{NAD}+$, has been recognized as a type of endogenous fluorophore. Beside the changes in fluorescence intensity of detected signal, advanced time resolved microscopy is able to sensitize increase/decrease of fluorescence signal decay in time (Drozdowicz-Tomsia et al. 2014). It is well known, that the lifetime of fluorescence emission is significantly affected by molecules surrounding the fluorophore. In general, very rapid fluorescence decay $(0.3 \mathrm{~ns})$ is characteristic for free (soluble) $\mathrm{NADH}$, while its protein bound species exhibit longer fluorescence lifetime (up to few nanoseconds), as summarized in Table 1. Based on this phenomenon, it is possible to distinguish the metabolic shift from glycolysis ( $0.3 \mathrm{~ns})$ to oxidative phosphorylation (2.5 ns) after binding to mitochondrial complex I (Skala et al. 2007). Conversely, inhibition of the terminal respiratory chain leads to glycolysis stimulation and soluble cytoplasmic NADH formation, which is accompanied by a rapid reduction in the fluorescence lifetime (Stringari et al. 2012). Accordingly, inhibition of terminal respiration in the mitochondria by rotenone is accompanied with switching of energy metabolism to favor anaerobic glycolysis. Such process then mimics the "Warburg effect" and usually results in shortening of NADH fluorescence lifetime. Majority of results using rotenone, were obtained after few minutes to hours of rotenone exposure (Blacker et al. 2014, Skala et al. 2007, Schneckenburger et al. 2004). However, NADH fluorescence lifetime changes in case of longer exposure ( $24 \mathrm{~h}$ and more) of rotenone have not been summarized yet.

Table 1. Possible $N A D(P) H$ fluorescence lifetime range in specific conditions detected by various authors.

\begin{tabular}{|c|c|c|c|}
\hline NAD(P)H species & \multicolumn{2}{|c|}{ Fluorescence lifetime } & Reference \\
\hline Free NADH (glycolysis) & \multicolumn{2}{|c|}{$\sim 0.3 \mathrm{~ns}$} & Skala et al. 2007 \\
\hline bound NADH (oxphos) & \multicolumn{2}{|c|}{$\sim 2.0-2.5 \mathrm{~ns}$} & Schneckenburger et al. 2004 \\
\hline bound NADPH & \multicolumn{2}{|c|}{$\sim 4 \mathrm{~ns}$} & Blacker et al. 2014 \\
\hline $\mathrm{NADH}+\alpha$ Syn monomeric & \multicolumn{2}{|c|}{$\sim$ free NADH } & \\
\hline $\mathrm{NADH}+\alpha$ Syn fibrils/bound to LDH & \multicolumn{2}{|c|}{$\sim>3.2 \mathrm{~ns}$} & Plotegher et al. 2015 \\
\hline NADH bound to LDH & \multicolumn{2}{|c|}{$\sim>3.2 \mathrm{~ns}$} & \\
\hline \multirow{2}{*}{ Short NADH lifetime } & $\begin{array}{l}\text { Intact } \\
\text { mitochondria }\end{array}$ & $0.4 \mathrm{~ns}$ & \multirow{4}{*}{ Blinova et al. 2005} \\
\hline & $\begin{array}{c}\text { Pulverized } \\
\text { mitochondria }\end{array}$ & $0.3 \mathrm{~ns}$ & \\
\hline \multirow[b]{2}{*}{ Long NADH lifetime } & $\begin{array}{c}\text { Intact } \\
\text { mitochondria }\end{array}$ & $5.7 \mathrm{~ns}$ & \\
\hline & $\begin{array}{l}\text { Pulverized } \\
\text { mitochondria }\end{array}$ & $4.1 \mathrm{~ns}$ & \\
\hline
\end{tabular}

According to a recent study by Plotegher (Plotegher et al. 2015), discrimination between long and short NADH lifetime fluorescence emission could also serve to monitor the process of protein aggregation in cells. In in vitro studies, the authors observed prolonged emission of $\mathrm{NADH}$ fluorescence in response to $\alpha$-synuclein overexpression or treatment with $\alpha$-synuclein protofibril seeds (Plotegher et al. 2015). This study demonstrated the potential for fluorescence lifetime imaging (FLIM) to be used as a simple and rapid monitoring tool for processes occurring at least in experimental models of PD.

Despite the large diagnostic potential of NADH fluorescence lifetime reported by Plotegher, there are still 
constraints, mainly due to lack of data on the specificity/localization of intracellular changes (cytoplasmic protein aggregation) and the NADH fluorescence lifetime analysis in vivo. Therefore, the aim of this work is to gain new knowledge on the possibilities as well as limitations of the NADH fluorescence lifetime analysis with respect to the monitoring of intracellular progression of PD in cellular model. Since it has not been answered yet, whether NADH binds directly to LB under pathological conditions or its accumulation occurs in parallel but at a different place in cell, the main objectives of this study are i) to specify and localize NADH lifetime changes on subcellular level in cells with PD-like pathogenesis ii) to gain knowledge of the specificity of $\mathrm{NADH}$ fluorescence lifetime probably associated with protein aggregates.

\section{Methods}

\section{Cell culture and rotenone treatment}

Human SH-SY5Y neuroblastoma cells (CRL-2266; ATCC, Manassas, VA, USA) were maintained in Dulbecco's modified Eagle's media (DMEM) supplemented with $10 \%$ fetal bovine serum (FBS, Biosera) and 1x penicillin/streptomycin (1x PS; Biosera) at $37{ }^{\circ} \mathrm{C}$ in a $5 \% \mathrm{CO}_{2}$ humidified atmosphere. For rotenone (Sigma, R8875) treatment, cells were seeded to plastic dishes (TPP, $9.2 \mathrm{~cm}^{2}$ growth surface) at concentration of 250,000 cells/plate. After $24 \mathrm{~h}$, the growth medium was changed to fresh DMEM supplemented with $10 \%$ FBS, 1x PS and treated with rotenone at final concentrations $10 \mathrm{nM}, 50 \mathrm{nM}$ and $100 \mathrm{nM}$. Final count of cells used for the analysis was set up to $2-5 \times 10^{6}$ cells per dish. Cells were imaged and analyzed $24 \mathrm{~h}$ after rotenone treatment. All procedures were repeated for at least 3 times and the most representative pictures were chosen for presentation.

\section{Immunocytochemistry}

For co-localization of $\alpha$-synuclein and $\beta I I I-t u b u l i n$, SH-SY5Y neuroblastoma cells were fixed in $4 \%$ paraformaldehyde (PFA), permeabilized in PBS with $0.1 \%$ Triton X-100, and blocked with PBS supplemented with $5 \%$ bovine serum albumin (BSA, Sigma). Cells were then processed for immunocytochemistry with primary mouse anti- $\alpha$-synuclein (Abcam, ab1903) and rabbit anti- $\beta$ III tubulin. The counterstaining was performed with anti-mouse IgG-AlexaFluor-488 Alexa (ThermoFisher scientific, A-11001) and goat anti-rabbit IgG AlexaFluor594 antibody (ThermoFisher scientific, A-11037) antibody. Cell nuclei were visualized with DAPI (Roche, 10236276001).

\section{Live cell staining}

The live cell staining was performed to visualize the mitochondria and for detection of the protein aggregation process. MitoTracker®RED FM (MTR, Invitrogen, M22425) was used to display the mitochondrial network with low specificity to the mitochondrial transmembrane potential. Alternatively, the tetramethylrhodamine (TMRM, Sigma, T5428) fluorescence probe was used to monitor transmembrane potential of mitochondria. Cells were stained in fresh growth medium containing MTR or TMRM at a final concentration of $100 \mathrm{nM}$ (100 mM stock in DMSO) for $30 \mathrm{~min}$ at $37^{\circ} \mathrm{C}$ in humidified $5 \% \mathrm{CO}_{2}$ atmosphere. Subsequently, the cells were washed three times with PBS. For regeneration, the cells were incubated for $30 \mathrm{~min}$ in fresh pre-warmed growth medium without dye at $37^{\circ} \mathrm{C}$ and immediately analyzed. The same staining conditions were maintained for detection of cytoplasmic inclusions in living cells by Thioflavine S (ThS, Sigma, $\mathrm{T} 1892$ ) with a final concentration of $0.0005 \% \mathrm{~m} / \mathrm{v}$. Naturally occurring endo-fluorophore NADH has also been used to monitor intracellular changes by confocal microscopy.

\section{Confocal microscopy}

Fluorescence images were acquired using a Carl Zeiss LSM 880 NLO (Carl Zeiss AG, Jena, Germany) multiphoton confocal imaging system equipped with multiple lasers $(405,488,561,594 \mathrm{~nm})$ and multiphoton laser Coherent Chameleon ULTRA II (680-1,080 nm, $3.5 \mathrm{~W}$ ) (Coherent Inc., Santa Clara, CA, USA). For the analysis of fixed cells we used Plan Apochromat 63x/1.4 Oil DIC objective was used. Living cells were analyzed using a W Plan Apochromat 40x/1.0 DIC objective. All confocal images were obtained using a bidirectional in frame scanning with pixel dwell about $4.0 \mu$ s and by maximum intensity projection of z-stacks composed by 10-15 focal planes of $1 \mu \mathrm{m}$ thickness.

One-photon excitation was used for imaging of ThS, NADH, Alexa-488 and 594, DAPI, MTR and TMRM. For two-photon excitation of NADH/ThS a fully tunable Ti: sapphire Chameleon ULTRA II (Coherent) set at $740 \mathrm{~nm}$ was used. Detection wavelengths were set by system automatically. The same confocal system 
parameters were retained for the entire set of images within one experimental group.

\section{Spectral analysis of overlapping emission wavelengths}

We used lambda mode to separate the fluorescence signal emitted by the fluorophores with near emission spectra (MTR/TMRM and NADH/ThS). The excitation wavelength used to visualize MTR/TMRM was $561 \mathrm{~nm}$. The emission maxima found for TMRM was $590 \mathrm{~nm}$ in comparison to $640 \mathrm{~nm}$ observed for MTR (Fig. 2C). Similarly, a narrow shift $(40-50 \mathrm{~nm})$ of fluorescence emitted by ThS (Emax $=430 \mathrm{~nm})$ was determined in contrast to the endogenous NADH signal $($ Emax $=480 \mathrm{~nm}) \quad$ (Fig. 4D). The emission spectrum differences were used to separate a specific signal by optional "zeiss signal unmixing" and visualized as colorcoded channels in relevant images.

\section{Fluorescence lifetime imaging (FLIM microscopy)}

FLIM microscopy was performed using a hybrid detector HPM-100-40 (Becker-Hickl, Germany) of light emission at 400-550 nm after excitation with two-photon chameleon laser at a wavelength of $740 \mathrm{~nm}$. Image acquisition was performed exclusively on living cells using W Plan Apochromat 40x/1.0 DIC objective, scanned as a single focal plane image, with one-directional in frame scanning mode in 512/512 pixel format and a pinhole opened in maximal range. Other settings remain unchanged compared to confocal imaging. Data for FLIM were acquired until at least 300 counts were collected in the brightest pixel of the image. As the acquisition software original Becker-Hickl SPC-150 was used. Analysis of the data was further performed by SPC image software (Becker-Hickl, Germany). Measured lifetime decay curve was binned over the pixel of interest and the 1 nearest neighbor pixel. Fluorescence lifetime was calculated from multi-exponential fits of emission decay curves. Survey of $\chi^{2}$ value indicated to use doubleexponential decay fit model for lifetime calculating. Quantum yield of short (a1) and long (a2) lifetime components were evaluated as a1/a2 ratio. Color range of images presented in Figure $1 \mathrm{~B}$ and $4 \mathrm{~B}$ is expressing average fluorescence lifetime. Color coding of FLIM images presented on Figure $2 \mathrm{~A}$ is based on values of a1/a2 ratio distribution.

\section{Statistical analysis of FLIM data}

The mean fluorescence lifetime values / a1 vs. a2 of the 10 regions of interest (ROIs) derived from the presented FLIM images were used. Statistical significance in the experiments was determined with respect to controls ( $0 \mathrm{nM}$ rotenone) with a one-way ANOVA. The results are graphically expressed as mean \pm SEM.

\section{Results}

Rotenone induces the intracellular accumulation of $\alpha$-synuclein and NADH in SH-SY5Y cells

From the start of experimental procedures, we have focused on cellular model validation in our conditions. In agreement with literature, we also confirmed by the immunocytochemical analysis that rotenone treatment $(50-100 \mathrm{nM}$ for $24 \mathrm{~h})$ leads to gradual accumulation of $\alpha$-synuclein in human SH-SY5Y cells in dose dependent manner (Fig. 1A). In addition, live cell analysis revealed the accumulation of the NADH fluorescence signal (Fig. 1B). Rotenone treatment for $24 \mathrm{~h}$ results in a significant reduction in NADH fluorescence lifetime, as is the evident from the histogram analysis of average NADH fluorescence lifetime (Fig. 1C), with the significant shift towards the shorter fluorescence lifetime in case on cells treated with $50 \mathrm{nM}$ and $100 \mathrm{nM}$ rotenone (Fig. 1D).

\section{Rotenone affects the mitochondrial transmembrane potential}

The main subcellular localization of NADH is associated with a mitochondrial matrix, with a certain amount of NADH of glycolytic origin also present in cytoplasm. To visualize the physiological status of the mitochondrial network in SH-SY5Y cells, we used i) MitoTracker®RED (MTR), a widely used molecular probe with low sensitivity to mitochondrial transmembrane potential and ii) TMRM probe that reflects the mitochondrial potential very well as it is necessary for TMRM transport into vital mitochondria. An increasing concentration of rotenone has a negative effect on the mitochondrial membrane potential. Treatment with 50-100 $\mathrm{nM}$ rotenone for $24 \mathrm{~h}$ results in complete loss of mitochondrial membrane potential in some cells, as indicated by staining of mitochondrial structures exclusively with MTR but not TMRM (Fig. 2A). MTR and TMRM have overlapping emission spectra, with the emission maximum at $595 \mathrm{~nm}$ and $645 \mathrm{~nm}$ respectively (Fig. 2B). The software spectral unmixing by lambda mode of image acquisition allowed us to analyze the whole mitochondrial network (MTR) and mitochondria with membrane potential (TMRM) separately (Fig. 2A). 
A

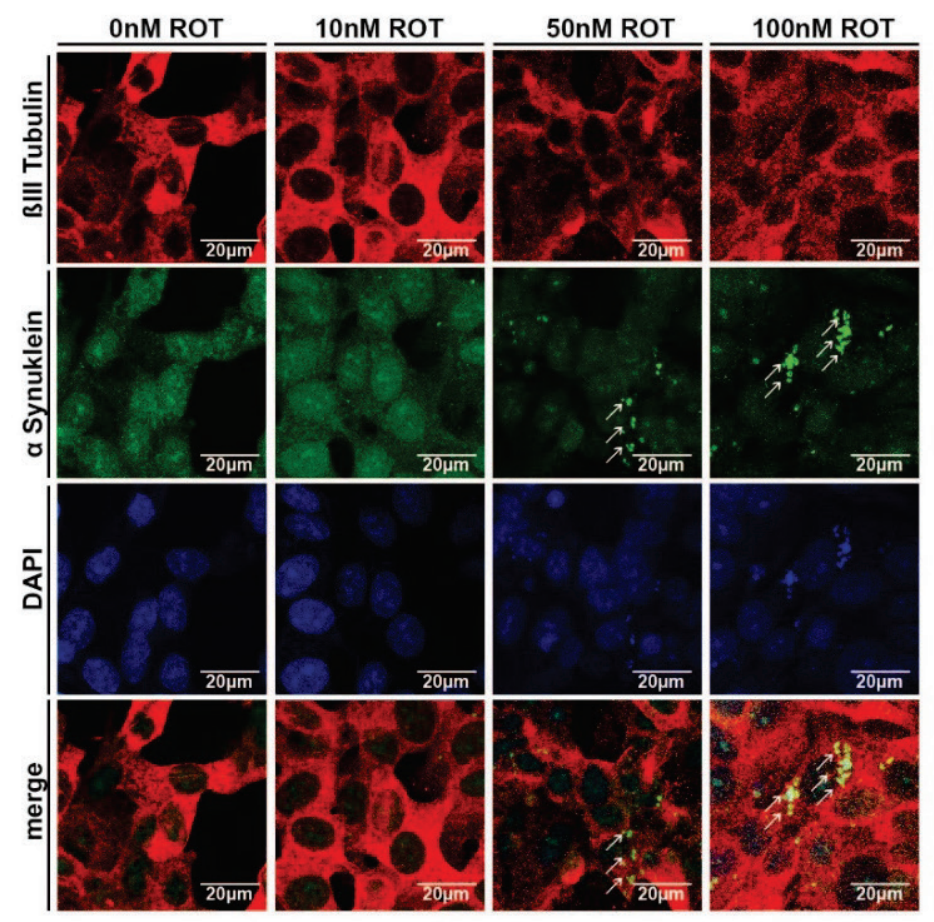

B

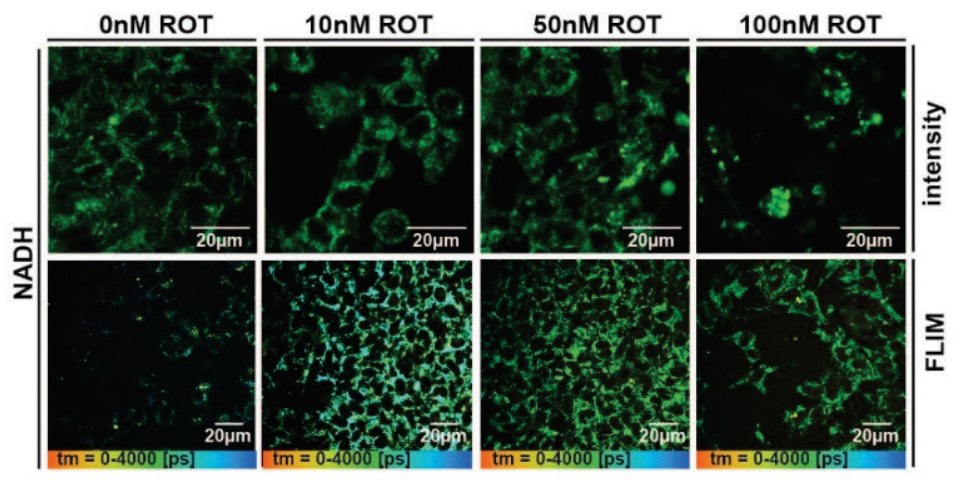

C

NADH Fluorescence lifetime

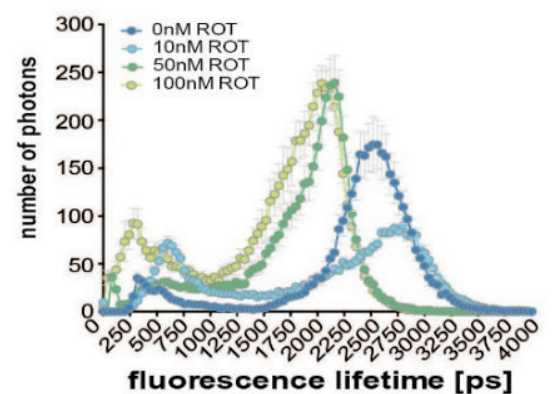

D

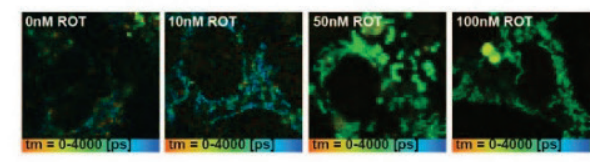

\begin{tabular}{lcccc} 
& OnM ROT & 10nM ROT & 50nM ROT & 100nM ROT \\
\hline MEDIAN [ps] & 2500 & 2660 & 2120 & 2040 \\
\hline min./max. & $2400 / 2720$ & $2600 / 2920$ & $2080 / 2200$ & $1920 / 2120$ \\
\hline significance & & ns & $* \star \star *$ & $* \star *$ \\
\hline
\end{tabular}

Fig. 1. Rotenone treatment induces intracellular accumulation of a-synuclein and NADH in SH-SY5Y cells. (A) SH-SY5Y cells treated with rotenone with increasing concentration for $24 \mathrm{~h}$, PFA fixed and stained for a-synuclein, BIII tubulin and DAPI and analyzed by confocal microscopy. Increased a-synuclein accumulation is observed in cells treated with $50 \mathrm{nM}$ or $100 \mathrm{nM}$ rotenone for $24 \mathrm{~h}$, whereas lower concentrations show no effect. Images are presented as maximum intensity projections of multiple z-stacks. (B) Confocal fluorescence imaging of live SH-SY5Y cells treated with rotenone for $24 \mathrm{~h}$. Accumulation of NADH is presented (top panel) with simultaneous acquisition of the fluorescence lifetime (lower panel) in rotenone dose dependent manner. Confocal images are presented as maximum intensity projections of multiple z-stacks, FLIM images represent one focal plane. (C) FLIM analysis of NADH fluorescence lifetime shift as a response to rotenone treatment (SEM, $n=10)$. (D) Statistical analysis and representative ROI of NADH fluorescence lifetime ( $n=10$, one-way ANOVA and multiple comparison test). 
A

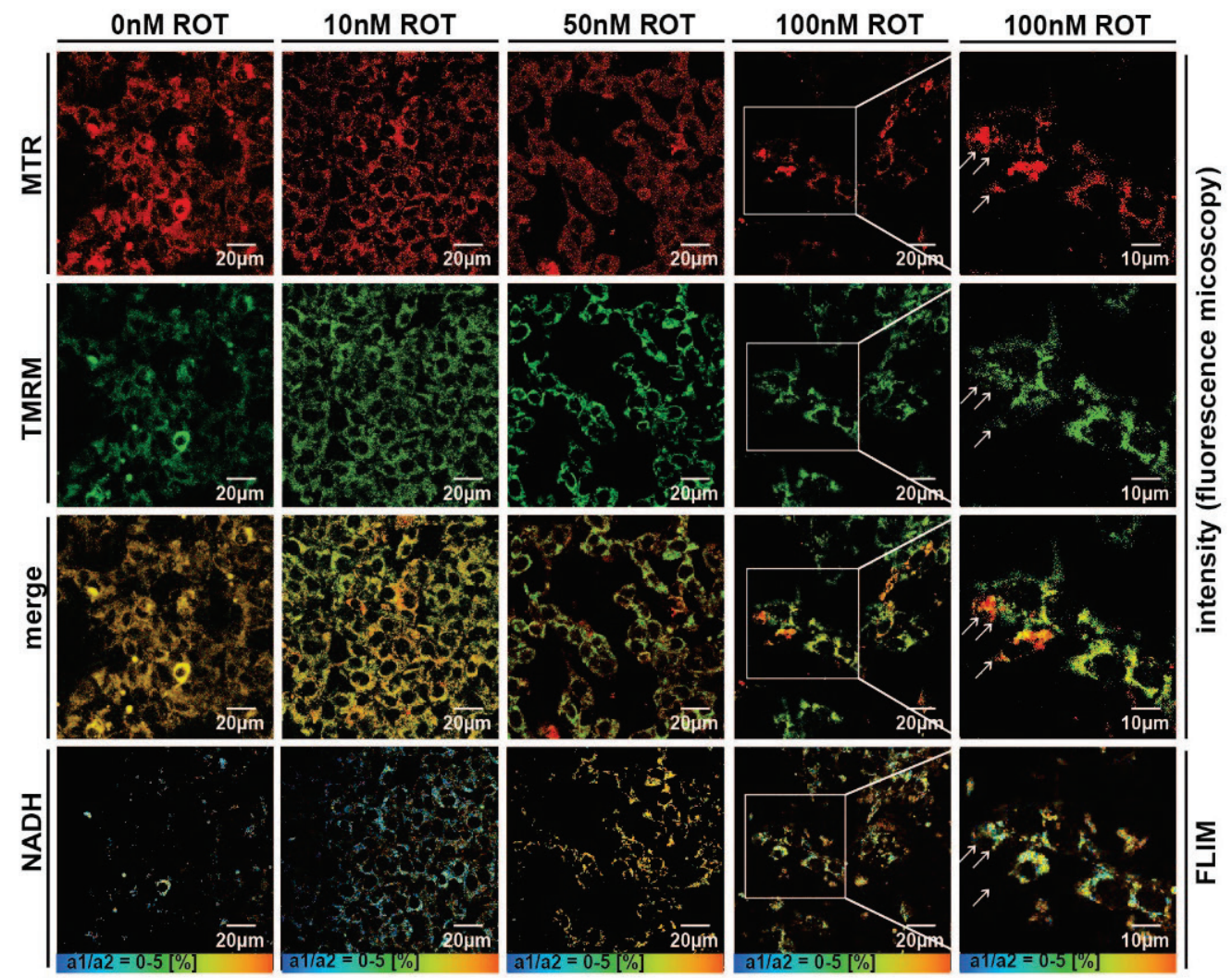

B

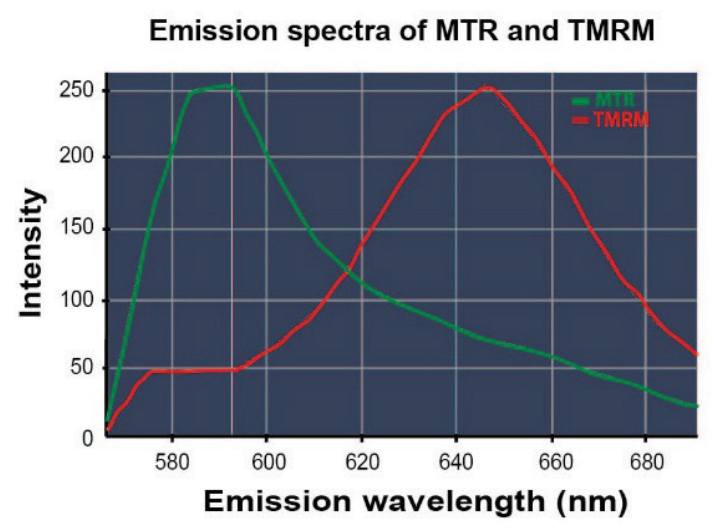

C

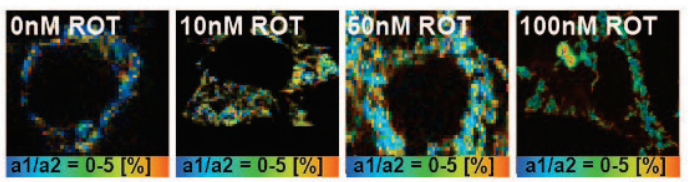

Proportional contribution of short (a1) and long (a2) NADH fluorescence lifetime components

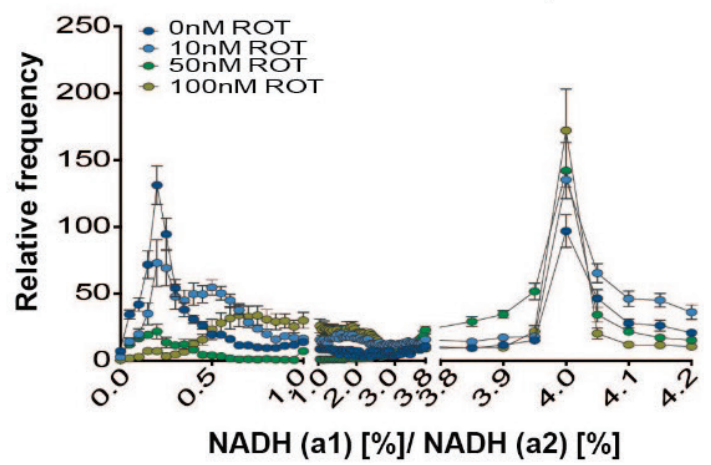

Fig. 2. Changes in the lifetime of $N A D H$ correlate with changes in the mitochondrial transmembrane potential. (A) Semi-quantitative evaluation of the mitochondrial transmembrane potential in living SH-SY5Y cells by TMRM, a mitochondrial potential-sensitive dye. The entire mitochondrial network is visualized using MTR (MitoTracker@RED). Increased rotenone concentration ( $24 \mathrm{~h}$ ) leads to loss of mitochondrial transmembrane potential (intensity). FLIM images of NADH are displayed as a ratio of short (a1) and long (a2) lifetime component of NADH fluorescence. Confocal images are presented as maximum intensity projections of multiple z-stacks, FLIM images represent one focal plane. (B) Emission maxima of TMRM and MTR used for signal separation in presented images. (C) Analysis of the prevalence of short (a1) and long (a2) components in cells treated with increased rotenone concentrations. Rotenone concentrationdependent continuous increase of the a1/a2 ratio reveal the shortening of the NADH fluorescence lifetime of NADH. 


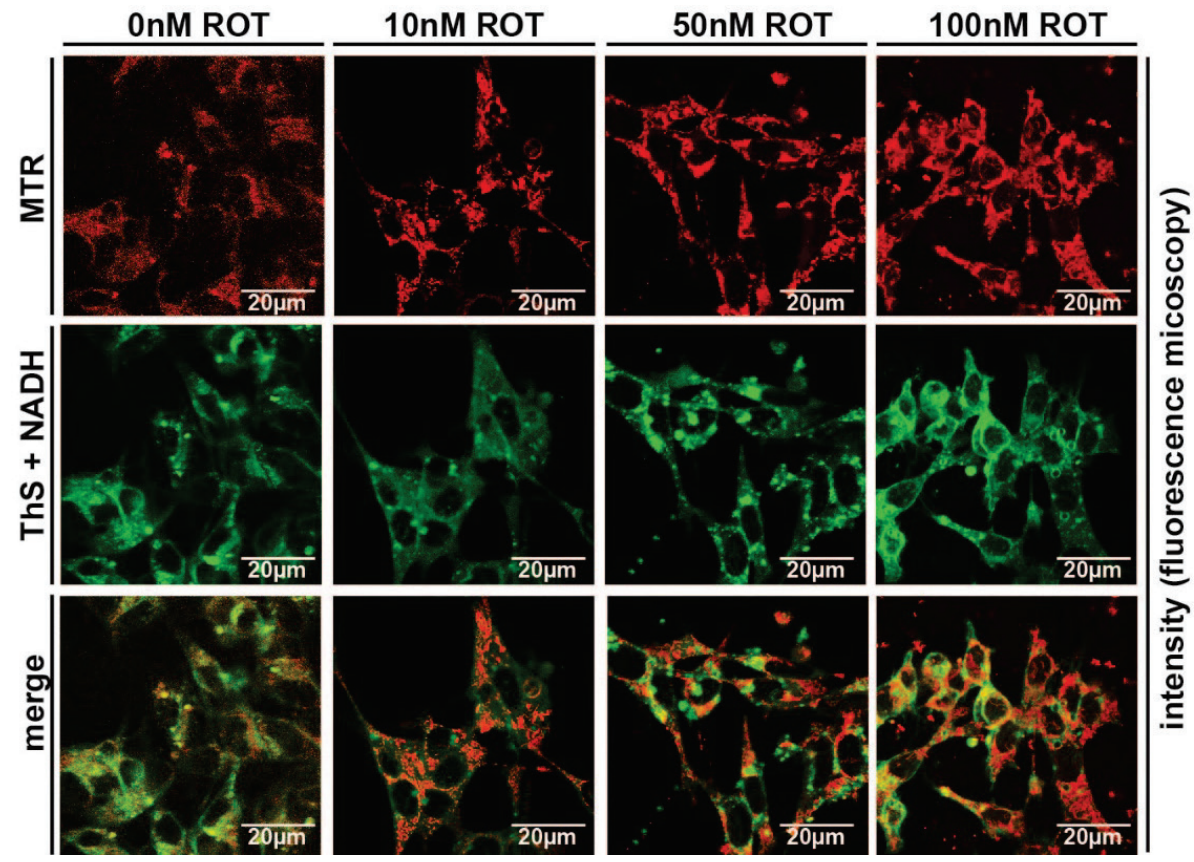

Fig. 3. Rotenone induces the accumulation of ThS and NADH. Confocal fluorescence imaging of live SH-SY5Y cells treated with rotenone $(24 \mathrm{~h})$ and co-stained with ThS and MTR. Higher concentration of rotenone ( $50 \mathrm{nM}$ and $100 \mathrm{nM})$ results in overall ThS accumulation in cellular structures, as well as in mitochondria. Signal of ThS is overlapped by NADH fluorescence in this image, as microscopic analysis was performed without spectral differentiation. Confocal images are presented as maximum intensity projections of multiple z-stacks.

As noted above, the lifetime of $\mathrm{NADH}$ fluorescence is significantly reduced in rotenone-treated cells. This effect can also be expressed as the ratio between the short (a1) and long (a2) NADH fluorescence long lifetime component. In the case of control cells $(0 \mathrm{nM}$ rotenone), blue color-coding indicates a more pronounced $\mathrm{NADH}$ fluorescence component with long lifetime (a2). For cells treated with $50-100 \mathrm{nM}$ rotenone, a clear shift towards the green spectrum where the NADH fluorescence component with a short lifetime (a1) prevails (Fig. 2C top panel). These results are displayed also graphically (Fig. 2C lower panel).

Rotenone induces the prolongation of ThS average fluorescence lifetime in SH-SY5Y cells

Further experiments were focused on the localization of the rotenone-induced cytoplasmic inclusions in relation to the mitochondrial network. Cells exposed to rotenone for $24 \mathrm{~h}$ were co-stained with $\mathrm{ThS}$, to detect the presence of protein inclusions and MTR, to distinguish the mitochondrial or non-mitochondrial origin of the fluorescence signal (Fig. 3). Structures containing the ThS fluorescence signal were detected both inside and outside the mitochondrial network. In addition, rotenone treatment (50-100 nM) led to an accumulation and increase in density of both MTR and ThS+NADH signal
(Fig. 3). Here we have detected mixed ThS and NADH fluorescence signal, due to their near emission spectra (Fig. 4D), further separation of this spectra is shown in Figure 4.

Further spectral analysis using the lambda mode of image acquisition was implemented in microscopic analysis that allowed us to separate the signals derived from ThS and NADH. In all samples we observed a strong co-localization of both, ThS and NADH, fluorescence signals. However, at higher concentrations of rotenone (50-100 nM), these two fluorophores are well separated at distinct structures in cells (Fig. 4A intensity). Moreover, these structures also significantly prolong the average lifetime of fluorescence of mixed $\mathrm{ThS}+\mathrm{NADH}$ fluorescence in these structures up to 2,000 ps (Fig. 4A FLIM). Due to the impossibility of applying lambda mode and spectral unmixing in FLIM analysis, we are not able to analyze the lifetime of fluorophores with near emission spectra separately. However, in combination with confocal microscopy, we are able to reveal that structures with prolonged average fluorescence lifetime (dark blue) are found in structures with a higher prevalence of NADH fluorescence signal compared to the ThS signal (Fig. 4A FLIM). The prolongation of the ThS+NADH fluorescence lifetime in rotenone concentration dependent manner, is also evident from the 
A

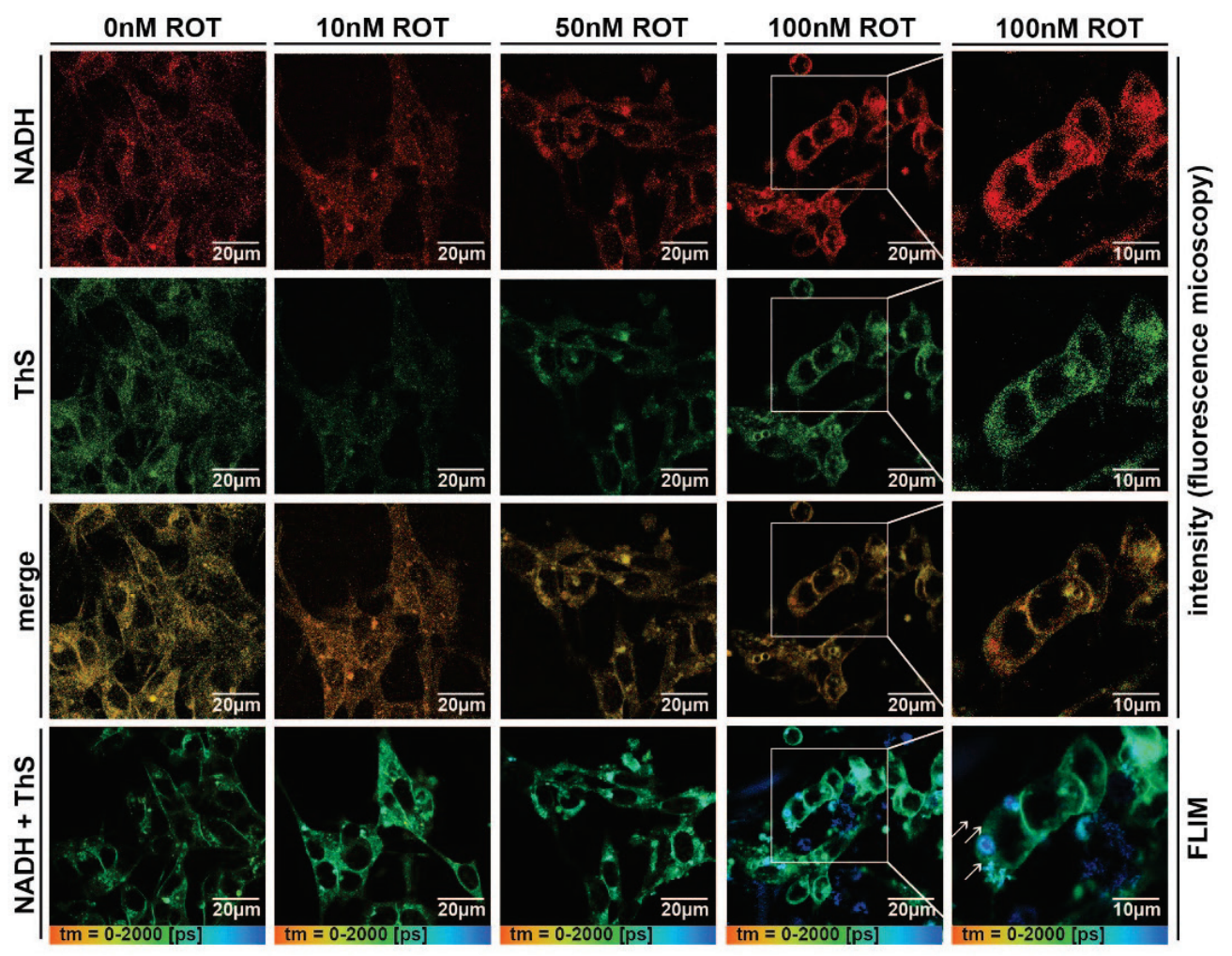

B

ThS + NADH Fluorescence lifetime

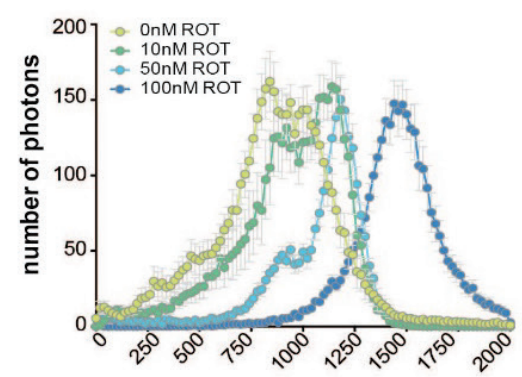

fluorescence lifetime [ps]

D

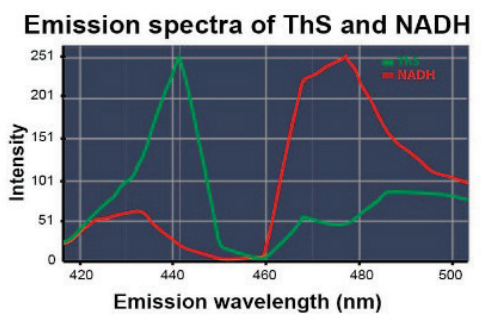

C

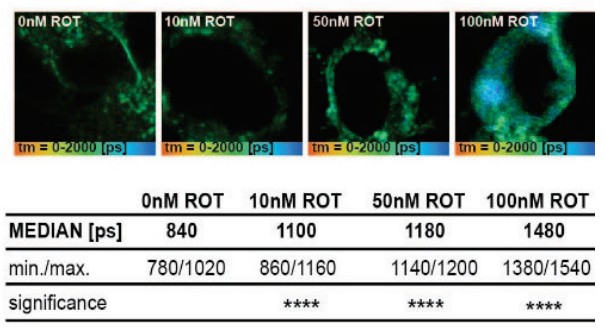

Fig. 4. Rotenone treatment induces the prolongation of ThS fluorescence lifetime in cells. (A) Confocal fluorescence microscopy (intensity) and FLIM analysis of live SH-SY5Y cells treated with rotenone ( $24 \mathrm{~h}$ ) and stained with ThS. Separation of ThS and NADH emission spectra was performed by the lambda mode. The differences in ThS and NADH density at the subcellular level are clearly visible at higher concentrations of rotenone $(50 \mathrm{nM}$ and $100 \mathrm{nM})$. Confocal images are supplemented with FLIMs of the one focal plane region to visualize the shift in the lifetimes of ThS and NADH in rotenone dose dependent manner. (B) FLIM analysis of ThS and NADH fluorescence lifetime shift as a response to rotenone treatment (SEM, $n=10)$. (C) Statistical analysis and representative ROI of the ThS and NADH fluorescence lifetime ( $n=10$, one-way ANOVA and multiple comparison test). (D) Emission maxima of NADH and ThS used for NADH/ThS signal separation in presented images. 
histograms of the mean fluorescence lifetime of the analyzed samples (Fig. 4B). The statistical analysis of ThS+NADH signal confirmed a significant shift towards the longer average fluorescence lifetime already at a $10 \mathrm{nM}$ rotenone concentration (Fig. 4C).

\section{Discussion}

In the present study, we provide additional data on localization of intracellular changes associated with PD pathogenesis as well as the possibility of using the FLIM analysis of the NADH and ThS fluorescence lifetime to identify these changes. The association between the protein aggregation processes and the NADH fluorescence lifetime has recently been described (Plotegher et al. 2015). In in vitro experiments using recombinant $\alpha$-synuclein, the authors provided evidence of differences in the NADH fluorescence lifetime depending on whether NADH is bound to enzymes or $\alpha$-synuclein protofibrils or is free in solution. Similarly, fluorescence lifetime fluctuation of NADH population after induction of protein aggregation by $\alpha$-synuclein seeds or $\alpha$-synuclein overexpression in HEK-293T cells has been detected. The enormous potential of NADH as a possible intracellular indicator of the protein aggregates formation has been underlined by this breakthrough study. Simultaneously with mentioned findings, relevant questions arose. The changes in NADH fluorescence lifetime have been analyzed without further co-localization of NADH with aggregated $\alpha$-synuclein. Subcellular localization of NADH with relevance to aggregated inclusions is also unknown. These questions remained to be answered.

Rotenone as a potent inhibitor of the mitochondrial respiratory complex I leads to mitochondrial function impairment (Lezi and Swerdlow 2012) and also acts as an inducer of protein aggregation and $\alpha$-synuclein overexpression (Blesa et al. 2012). Accumulation of $\alpha$-synuclein in parallel with accumulation of NADH in rotenone treated cells was also confirmed by our results (Fig. 1A, B). Moreover, published data obtained after short-term (minutes to hours) exposure to rotenone indicate a clearly shift in $\mathrm{NADH}$ fluorescence towards the shorter lifetimes relevant to free (cytoplasmic) NADH. This phenomenon is explained by the inhibition of terminal oxidation in mitochondria and prevalent energy production by substrate phosphorylation in anaerobic glycolysis (Blacker et al. 2014, Skala et al. 2007, Schneckerburger et al. 2004). Our results of the NADH fluorescence lifetime analysis confirm the shift in NADH fluorescence lifetime towards the shorter intervals even after $24 \mathrm{~h}$ of rotenone exposure (Fig. 1C, D). This phenomenon is further confirmed by analysis of the ratio of short (a1) and long (a2) lifetime components (Fig. 2B, C).

In addition to NADH fluorescence lifetime analysis, we also focused on determining the physiological state of the mitochondrial network after $24 \mathrm{~h}$ of cell exposure to rotenone. To visualize mitochondrial density, we used a MitoTracker®RED probe. TMRM, a transmembrane marker of potential, was used to detect changes in transmembrane electrogradient in cells exposed to increasing concentrations of rotenone for $24 \mathrm{~h}$. By MTR and TMRM spectral unmixing, we observed that a higher dose of rotenone (50-100 nM) induced a clear distribution of MTR and TMRM signal in distinct regions of the mitochondrial network (Fig. 2A). The leakage and fluorescence intensity decay of TMRM was previously confirmed by different research teams in different cellular models after short-term exposure of higher rotenone doses $(5 \mu \mathrm{M})$ (Emmrich et al. 2013, Gyulkhandanyan et al. 2003).

One of the limitations of the current study is the absence of phasor plot analysis. Discrimination between small fluorescence lifetime shifts could become invisible in a continuous range application. The absence of the software we solved by the ThS staining, a widely used specific indicator of protein aggregation in living cells. ThS staining of the cells indicated a higher fluorescence density particularly in samples treated with 50-100 nM rotenone for $24 \mathrm{~h}$ (Fig. 3). Partial co-localization of ThS and MTR signals also suggests possible mitochondrial localization of protein aggregates in rotenone-treated cells. Mitochondrial localization of $\alpha$-synuclein, as well as its functional impact on terminal respiratory chain has been recently described by Ludtmann et al. (2018).

In case of detection of $\mathrm{ThS}$ fluorescence in living cells, this is accompanied by the fluorescence derived from NADH due to the near emission spectra of both fluorophores. Following NADH and ThS spectral unmixing by lambda mode (Fig. 4A, D), the strong ThS and NADH co-localization indicates the presence of $\mathrm{NADH}$ molecules in close proximity to ThS-positive protein aggregates. At higher concentration of rotenone (50-100 nM), the distinct NADH-positive structures with lower ThS density were detected. Origin and content of these structures remains to be investigated. However, the mean fluorescence lifetime of the ThS/NADH signal in 
rotenone-treated cells determined the importance of the rotenone effect in favor of prolonging the fluorescence lifetime in the ThS stained samples (Fig. 4).

The results obtained by ThS staining showed the opposite effect of rotenone on the mean fluorescence lifetime in case of mixed $\mathrm{ThS}+\mathrm{NADH}$ signal, compared when a fluorescence signal from NADH alone was detected. This indicates specific contribution of $\mathrm{ThS}$ fluorescence towards a longer fluorescence lifetimes. Binding of amyloid-specific Thioflavin $\mathrm{T}$ to amyloid fibrils as described by Lindberg et al. (2015) similarly showed the prolongation of the Thioflavin $\mathrm{T}$ fluorescence lifetime. The prolonged time interval was directly proportional to the length of the amyloid fibril in the study. Current ThS fluorescence lifetime results represent the first and original data obtained in vivo with the $\mathrm{S}$ type of thioflavin probe.

In conclusion, our findings provide relevant additional data to those discussed by Plotegher et al. (2015). Co-localization of ThS fluorescence signal with those of mitochondria (MTR) and NADH suggests a common topological localization of part of the protein aggregates within the cell in PD model. In addition, the shift of the ThS fluorescence lifetime towards the longer time intervals after rotenone treatment present the original and novel findings that contribute to the possible development of a sensitive methodological approach for the detection of the early changes in living cells associated with the pathogenesis of Parkinson's disease.

\section{Conflict of Interest}

There is no conflict of interest.

\section{Acknowledgements}

This publication was supported by APVV-15-0163 and VEGA 1/0334/18.

\section{References}

BLACKER TS, DUCHEN MR: Investigating mitochondrial redox state using NADH and NADPH autofluorescence. Free Radic Biol Med 100: 53-65, 2016.

BLESA J, PHANI S, JACKSON-LEWIS V, PRZEDBORSKI S: Classic and new animal models of Parkinson's disease. J Biomed Biotechnol 2012: 845618, 2012.

BLESA J, PRZEDBORSKI S: Parkinson's disease: animal models and dopaminergic cell vulnerability. Front Neuroanat 8: 155, 2014.

BLINOVA K, CARROLL S, BOSE S, SMIRNOV AV, HARVEY JJ, KNUTSON JR, BALABAN RS: Distribution of mitochondrial NADH fluorescence lifetimes: steady-state kinetics of matrix NADH interactions. Biochemistry 44: 2585-2594, 2005.

BRAAK H, DEL TREDICI K, RUB U, DE VOS RA, JANSEN STEUR EN, BRAAK E: Staging of brain pathology related to sporadic Parkinson's disease. Neurobiol Aging 24: 197-211, 2003.

CHOUCHANI ET, PELL VR, GAUDE E, AKSENTIJEVIC D, SUNDIER SY, ROBB EL, LOGAN A, NADTOCHIY SM, ORD ENJ, SMITH AC, EYASSU F, SHIRLEY R, HU CH, DARE AJ, JAMES AM, ROGATTI S, HARTLEY RC, EATON S, COSTA ASH, BROOKES PS, DAVIDSON SM, DUCHEN MR, SAEB-PARSY K, SHATTOCK MJ, ROBINSON AJ, WORK LM, FREZZA C, KRIEG T, MURPHY MP: Ischaemic accumulation of succinate controls reperfusion injury through mitochondrial ROS. Nature 515: 431-435, 2014.

DEVI L, RAGHAVENDRAN V, PRABHU BM, AVADHANI NG, ANANDATHEERTHAVARADA HK: Mitochondrial import and accumulation of alpha-synuclein impair complex I in human dopaminergic neuronal cultures and Parkinson disease brain. J Biol Chem 283: 9089-9100, 2008.

DICKSON DW, FUJISHIRO H, ORR C, DELLEDONNE A, JOSEPHS KA, FRIGERIO R, BURNETT M, PARISI JE, KLOS KJ, AHLSKOG JE: Neuropathology of non-motor features of Parkinson disease. Parkinsonism Relat Disord 15 (Suppl 3): S1-S5, 2009.

DROZDOWICZ-TOMSIA K, ANWER AG, CAHILL MA, MADLUM KN, MAKI AM, BAKER MS, GOLDYS EM: Multiphoton fluorescence lifetime imaging microscopy reveals free-to-bound NADH ratio changes associated with metabolic inhibition. J Biomed Opt 19: 086016, 2014.

EMMRICH JV, HORNIK TC, NEHER JJ, BROWN GC: Rotenone induces neuronal death by microglial phagocytosis of neurons FEBS J 280: 5030-5038, 2013. 
GYULKHANDANYAN AV, FEENEY CJ, PENNEFATHER PS: Modulation of mitochondrial membrane potential and reactive oxygen species production by copper in astrocytes. J Neurochem 87: 448-460, 2003.

HISAHARA S, SHIMOHAMA S: Toxin-induced and genetic animal models of Parkinson's disease. Parkinsons Dis 2011: 951709, 2010.

IYER A, PETERSEN NO, CLAESSENS MM, SUBRAMANIAM V: Amyloids of alpha-synuclein affect the structure and dynamics of supported lipid bilayers. Biophys $J$ 106: 2585-2594, 2014.

LEZI E, SWERDLOW RH: Mitochondria in neurodegeneration. Adv Exp Med Biol 942: 269-286, 2012.

LINDBERG DJ, WRANNE MS, GILBERT GATTY M, WESTERLUND F, ESBJORNER EK: Steady-state and timeresolved Thioflavin-T fluorescence can report on morphological differences in amyloid fibrils formed by Abeta(1-40) and Abeta(1-42). Biochem Biophys Res Commun 458: 418-423, 2015.

LUDTMANN MHR, ANGELOVA PR, HORROCKS MH, CHOI ML, RODRIGUES A, BAEV AY, BEREZHNOV AV, YAO Z, LITTLE D, BANUSHI B, AL-MENHALI AS, RANASINGHE RT, WHITEN DR, YAPOM R, DOLT KS, DEVINE MJ, GISSE P, KUNATH T, JAGANJAC M, PAVLOV EV, KLENERMAN D, ABRAMOV AY, GANDHI S: $\alpha$-synuclein oligomers interact with ATP synthase and open the permeability transition pore in Parkinson's disease. Nat Commun 9: 2293, 2018.

NAKAMURA K, MORI F, TANJI K, MIKI Y, TOYOSHIMA Y, KAKITA A, TAKAHASHI H, YAMADA M, WAKABAYASHI K: alpha-Synuclein pathology in the cranial and spinal nerves in Lewy body disease. Neuropathology 36: 262-269, 2016.

NAKAMURA K, NEMANI VM, AZARBAL F, SKIBINSKI G, LEVY JM, EGAMI K, MUNISHKINA L, ZHANG J, GARDNER B, WAKABAYASHI J, SESAKI H, CHENG Y, FINKBEINER S, NUSSBAUM RL, MASLIAH E, EDWARDS RH: Direct membrane association drives mitochondrial fission by the Parkinson diseaseassociated protein alpha-synuclein. J Biol Chem 286: 20710-20726, 2011.

NAKAMURA K, NEMANI VM, WALLENDER EK, KAEHLCKE K, OTT M, EDWARDS RH: Optical reporters for the conformation of alpha-synuclein reveal a specific interaction with mitochondria. $J$ Neurosci 28: 12305-12317, 2008.

PAN-MONTOJO F, ANICHTCHIK O, DENING Y, KNELS L, PURSCHE S, JUNG R, JACKSON S, GILLE G, SPILLANTINI MG, REICHMANN H, FUNK RH: Progression of Parkinson's disease pathology is reproduced by intragastric administration of rotenone in mice. PLoS One 5: e8762, 2010.

PAPÁČKOVÁ Z, CAHOVÁ Z: Important role of autophagy in regulation of metabolic processes in health, disease and aging. Physiol Res 63: 409-420, 2014.

PLOTEGHER N, STRINGARI C, JAHID S, VERONESI M, GIROTTO S, GRATTON E, BUBACCO L: NADH fluorescence lifetime is an endogenous reporter of alpha-synuclein aggregation in live cells. FASEB $J$ 29: 2484-2494, 2015.

SIMUNI T, SETHI K: Nonmotor manifestations of Parkinson's disease. Ann Neurol 64 (Suppl 2): S65-S80, 2008.

SKALA MC, RICHING KM, GENDRON-FITZPATRICK A, EICKHOFF J, ELICEIRI KW, WHITE JG, RAMANUJAM N: In vivo multiphoton microscopy of NADH and FAD redox states, fluorescence lifetimes, and cellular morphology in precancerous epithelia. Proc Natl Acad Sci U S A 104: 19494-19499, 2007.

SCHNECKENBURGER H, WAGNER M, WEBER P, STRAUSS WS, SAILER R: Autofluorescence lifetime imaging of cultivated cells using a UV picosecond laser diode. J Fluoresc 14: 649-654, 2004.

STRINGARI C, NOURSE JL, FLANAGAN LA, GRATTON E: Phasor fluorescence lifetime microscopy of free and protein-bound NADH reveals neural stem cell differentiation potential. PLoS One 7: e48014, 2012.

VOKURKOVÁ M, RAUCHOVÁ H, ŘEZÁČOVÁ L, VANĚČKOVÁ I, ZICHA J: NADPH oxidase activity and reactive oxygen species production in brain and kidney of adult male hypertensive Ren-2 transgenic rats. Physiol Res 64: 849-856, 2015.

WONG YC, KRAINC D: $\alpha$-synuclein toxicity in neurodegeneration: mechanism and therapeutic strategies. Nat Med 23: 1-13, 2017.

XU HN, ZHENG G, TCHOU J, NIOKA S, LI LZ: Characterizing the metabolic heterogeneity in human breast cancer xenografts by 3D high resolution fluorescence imaging. Springerplus 2: 73, 2013. 